\title{
The effect of ribosomal protein S15a in lung adenocarcinoma
}

Yifan Zhang, Guangxin Zhang, Xin Li, Bingjin Li, Xingyi Zhang

Background: RPS15A (ribosomal protein S15A) promotes mRNA/ribosome interactions in translation . It is critical for the process of eukaryotic protein biosynthesis. Recently, aberrantly expressed RPS15A was found in the virus hepatitis and malignant tumors. However, the role of RPS15A has not been fully revealed on the development of lung cancer. Method: In this study, a tissue microarray (TMA) of primary lung adenocarcinoma tissue specimens was carried out. Furthermore, to further investigate the function of RPS15A in lung cancer, RPS15A-specific short hairpin RNA (shRNA) expressing lentivirus (LV-shRPS15A) was constructed and used to infect H1299 and A549 cells. Result: Our data showed that RPS15A expression was increase in tumor tissues. Furthermore, the knockdown of RSP15A inhibited cancer cell growth and induced apoptosis in the cancer cells. Gene expression profile microarray also revealed that the P53 signaling pathway was activated in LV-shRPS15A-infected cancer cells. Conclusion: Taken together, our results demonstrate that RPS15A is a novel oncogene in non-small cell lung cancer and may be a potential therapeutic target in lung cancer. 
2

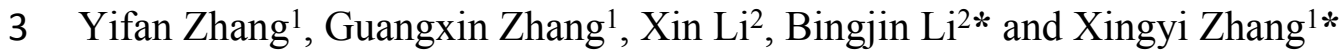

$4{ }^{1}$ Department of Thoracic Surgery, the Second Hospital of Jilin University, Changchun, China $5 \quad 130041$

$6{ }^{2}$ Jilin provincial key laboratory on molecular and chemical genetic, the Second Hospital of Jilin

7 University, Changchun, China 130041

8

9

10

11

\section{$12 *$ Corresponding author}

13 Xingyi Zhang,

14 Department of Thoracic Surgery

15 The Second Hospital of Jilin University

16218 Ziqiang street Changchun China 130041

17 Email: xyzhang@jlu.edu.cn

18

19 Bingjin Li,

20 Jilin provincial key laboratory on molecular and chemical genetic

21 The Second Hospital of Jilin University

22218 Ziqiang street Changchun China 130041

23 Email: bingjinli@hotmail.com 


\section{Abstract}

25 Background: RPS15A (ribosomal protein S15A) promotes mRNA/ribosome interactions in 26 translation. It is critical for the process of eukaryotic protein biosynthesis. Recently, aberrantly 27 expressed RPS15A was found in the virus hepatitis and malignant tumors. However, the role of 28 RPS15A has not been fully revealed on the development of lung cancer.

29 Method: In this study, a tissue microarray (TMA) of primary lung adenocarcinoma tissue 30 specimens was carried out. Furthermore, to further investigate the function of RPS15A in lung 31 cancer, RPS15A-specific short hairpin RNA (shRNA) expressing lentivirus (Lv-shRPS15A) was 32 constructed and used to infect H1299 and A549 cells.

33 Result: Our data showed that RPS15A expression was increase in tumor tissues. Furthermore, 34 the knockdown of RSP15A inhibited cancer cell growth and induced apoptosis in the cancer cells. 35 Gene expression profile microarray also revealed that the P53 signaling pathway was activated in 36 LV-shRPS15A-infected cancer cells.

37 Conclusion: Taken together, our results demonstrate that RPS15A is a novel oncogene in non38 small cell lung cancer and may be a potential therapeutic target in lung cancer. 
40

41

42

43

44

45

46

47

48

49

50

51

52

53

54

55

56

57

58

59

60

61

62

63

64

65

66

67

68

69

70

71

72

73

74

75

76

\section{Introduction}

Lung cancer is the most prevalent malignant tumor and the leading cause of cancer-related death in the world (Jemal et al. 2011). The principal histological forms of lung cancer have been well established. Approximately $85 \%$ of lung cancer cases are non-small cell lung cancer (NSCLC). Adenocarcinoma is the most commonly diagnosed type (Butnor \& Beasley 2007). Despite advances in surgical techniques and other therapeutic strategies, most patients diagnosed with lung cancer ultimately succumb to this disease within 5 years (Miller 2005; Paleari et al. 2008). Therefore, comprehensive understanding of the molecular mechanisms underlying lung cancer progress is important for the development of optimal anti-cancer therapy (Hensing et al. 2014).

Tumorigenesis involves multistep development to acquire certain malignant capabilities, such as, sustaining proliferative signaling, resisting cell apoptosis, activating invasion and metastasis et al (Hanahan \& Weinberg 2011). Underlying these abilities of tumor cells, rapid de novo biosynthesis of functional ribosome is essential for cancer cells to aggressively grow and obtain multiple malignant phenotypes. Ribosomes are composed of diverse ribosomal RNAs in eukaryotic organs. So far, most ribosomal proteins have been identified to bind to certain regions of ribosomal RNA and perform catalytic functions. Many ribosomal proteins have various extraribosomal functions, such as DNA repair, transformation, development, apoptosis, and transcription (Lee et al. 2010; Nishiura et al. 2013; Nosrati et al. 2014). Some studies show that these proteins may play a role in human tumor development and progression. For example, depletion of ribosomal protein L26 and L29 suppress the proliferation of human pancreatic cancer PANC-1 cells (Li et al. 2012), while RPL22 expression is highly associated with NSCLC (Yang et al. 2013).

Human ribosomal protein S15a (RPS15A) is a highly conserved cellular gene that maps to human chromosome 16p12.3 locus (Chan et al. 1994; Schaap et al. 1995). It promotes mRNA/ribosome binding in translation via the interactions with the cap-binding subunit of eukaryotic initiation factor 4F (eIF-4F) (Linder \& Prat 1990). In yeast, G1/S cell cycle phase arrest induced by $c d c 33$ (encoding eIF-4F in yeast) mutation could be reversed by RPS15A overexpression, these findings suggest that RPS15A may play a role in cell cycle transition (Lavoie et al. 1994). RPS15A over-expression also facilitates hepatocellular growth via promoting cell cycle transition and accelerates tumor formation in vitro (Lian et al. 2004), whereas RPS15A mRNA down-regulation inhibited hepatic cancer cell growth (Xu et al. 2014). However, the role of RPS15A in lung cancer has not been completely studied.

76 The present study was aimed to investigate whether RPS15A is involved in the development and 
77 progression of lung cancer. A tissue microarray was carried out to determine the expression of 78 RPS15A. To examine its functional role in lung cancer progress, an RPS15A-specific small 79 interfering RNA (siRNA)-lentiviral vector was constructed to block RPS15A expression in 80 human lung adenocarcinoma H1299 and A549 cells. Furthermore, the impacts of RPS15A 81 silencing on the growth of the cancer cells were examined by MTT assay and colony formation 82 assay. In addition, the effects of RPS15A knockdown on the apoptosis of H1299 and A549 cells 83 were determined by flow cytometry analysis. To further explore the potential molecular 84 mechanisms, we also performed a human whole genome oligo microarray followed by a KEGG 85 pathway enrichment analysis.

86 
89

90

91

92

93

94

95

96

97

98

99

100

101

102

103

104

105

106

107

108

109

110

111

112

113

114

115

116

117

118

119

120

121

122

123

124

\section{Materials and methods}

\section{Tissue microarray and immunohistochemical staining}

Tumor samples were collected from 75 patients with lung adenocarcinoma at the Department of Thoracic Surgery, The Second Affiliated Hospital of Jilin University, China, from July 2005 to December 2011. All the tissue samples were obtained from surgery with informed consent and with institutional review board approval of the hospital. Non-tumor samples from the macroscopic tumor margin were isolated at the same time and used as the matched adjacent nonneoplastic tissues. Expression of RPS15A protein was detected using immunohistochemical analysis on commercially available tissue microarrays (TMAs) from Shanghai Zhuoli Biotechnology Co., Ltd. (Shanghai, China), which contained a total of 150 tissue samples of tumor or adjacent normal tissues from 75 patients. There were 44 male patients and 31 female patients aging from 32 to 80 years, with an average age of 58 years old. The tumors were classified according to the tumor nodes metastasis (TNM) stage revised by the International Union Against Cancer in 2002.

\section{Immunohistochemical staining and scoring}

To detect RPS15A expression in lung cancer, archival paraffin-embedded tumor samples were used to build up tissue microarray (TMA) blocks for immunohistochemical (IHC) staining. Immunohistochemical staining was performed using the Vectastain Elite ABC Kit (Vector Laboratories, Burlingame, CA) according to the manufacturer's protocol. Briefly, TMA sections were deparaffinized and hydrated in xylene, ethanol and water. After heat-induced antigen retrieval procedures, sections were incubated overnight at $4{ }^{\circ} \mathrm{C}$ with anti-RPS15A primary antibody (1:50 dilution; Abcam). After the primary antibody was washed off, the ABC detection system was performed by using biotinylated anti-rabbit IgG. The slides were counterstained with haematoxylin and mounted in xylene mounting medium for examination. Negative controls were treated identically but with the primary antibody omitted. Three researchers evaluated immunoreactivity independently. The percentage of positive tumor cells was determined by each observer, and the average of three scores was calculated. The proportion of immunopositive cells were categorized as following: intensity of staining: none (0), mild (1), moderate (2), strong (3); percentage of the positive staining: $0(-),<15 \%(+), 15-50 \%(++),>50 \%(+++)$. To obtain final statistical results, - and + groups were considered as negative.

\section{Cell lines}


125 Lung adenocarcinoma cell lines H1299 and A549, lung squamous cancer cell line SK-MES-1, as 126 well as small cell lung cancer cell line H1688 (Cell Bank of Chinese Academy of Sciences, 127 Shanghai, China) and human embryonic kidney (HEK) 293T cell line (American Type Culture 128 Collection, ATCC, Manassas, VA, USA) were maintained in DMEM (Hyclone, Logan, UT, 129 USA) with 10\% FBS (Hyclone) and penicillin/streptomycin at $37^{\circ} \mathrm{C}$ in humidified atmosphere of $1305 \% \mathrm{CO}_{2}$.

131

132 133

134

135

136

137

138

139

140

141

142

143

144

145

146

147

148

149

150

151

152

153

154

155

156

157

158

159

160

161

\section{Construction and infection of RPS15A short hairpin (shRNA)-expressing lentivirus}

To permit robust inducible RNAi-mediated RPS15A silencing in tumor cells, RPS15A-specific shRNA containing lentiviral vector was constructed. The RNAi was designed based on conservative cDNA fragments within the coding region of human RPS15A gene (NM_001019)targeting sequence (5'-GCAACTCAAAGACCTGGAA -3') of oligo nucleotides. The sequences were annealed and ligated into the Age I/EcoR I (NEB, Ipswich, MA, USA)-linearized pGCSILGFP vector (Shanghai Genechem Co. LTD., Shanghai, China). The lentiviral-based shRNAexpressing vectors were confirmed by DNA sequencing. Recombinant lentiviral vectors and packaging vectors were then cotransfected into 293T cells using Lipofectamine 2000 (Invitrogen, Carlsbad, CA, USA), according to the manufacturer's instructions for the generation of recombinant lentiviruses Lv-shRPS15A and negative control Lv-shCon. The culture supernatants containing lentiviral particles expressing Lv-shRPS15A and Lv-shCon were harvested and ultracentrifuged $48 \mathrm{~h}$ after transfection, respectively. H1299 and A549 cells were infected with the lentiviruses at multiplicity of infection (MOI) of 10 and 20, respectively.

\section{Quantitative real-time PCR analysis}

In brief, total RNA was extracted using TRIzol reagent (Invitrogen, Carlsbad, CA). The reverse transcription reactions were carried out following the protocol of the M-MLV Reverse Transcriptase (Promega Corp., Madison, WI). Real-time quantitative PCR analysis was performed using SYBR Master Mixture Kit (TaKaRa, Dalian, China). The primer sequences for PCR amplification of RPS15A were 5'-CTCCAAAGTCATCGTCCGGTT-3' and 5'TGAGTTGCACGTCAAATCTGG-3'. GAPDH was applied as an internal control. The primer sequences of GAPDH were 5'-TGACTTCAACAGCGACACCCA-3'and 5'CACCCTGTTGCTGTAGCCAAA-3'. 2- $2^{-\Delta C T}$ method was adopted to calculate the relative expression levels of RPS15A by subtracting CT values of the control gene from the CT values of RPS15A.

\section{MTT proliferation and colony formation assay}


162

163

164

165

166

167

168

169

170

171

172

173

174

175

176

177

178

179

180

181

182

183

184

185

186

187

188

189

190

191

192

193

194

195

196

197

198

Briefly, exponentially growing cancer cells were inoculated into 96 -well plates with $2 \times 10^{4}$ cells per well. After incubation for 24, 48, 72, 96 and $120 \mathrm{~h}, 10 \mu \mathrm{l}$ of sterile MTT $(5 \mathrm{mg} / \mathrm{ml})$ was added into each well. Following incubation at $37^{\circ} \mathrm{C}$ for $4 \mathrm{~h}$, the reaction was blocked by adding $100 \mu 1$ of dimethyl sulfoxide. The formazan production was determined by measurement of the spectrometric absorbance at $490 \mathrm{~nm}$. The values obtained are proportional to the amount of viable cells and each experiment was repeated three times. In colony formation assay, H1299 and A549 cells infected with Lv-shRPS15A or Lv-shCon were seeded in six-well plates with $5 \times 10^{2}$ cells per well and cultured at $37^{\circ} \mathrm{C}$ with $5 \% \mathrm{CO}_{2}$ for 14 days. The cell colonies were washed twice with PBS, fixed in 4\% paraformaldehyde for $30 \mathrm{~min}$ and stained with Giemsa for $20 \mathrm{~min}$. Individual colonies with more than 50 cells were counted under a fluorescence microscope.

\section{Apoptosis assay by fluorescence-activated cell sorting (FACS) analysis}

In apoptotic cell detection, exponentially growing H1299 and A549 cells were seeded in six-well plates. After $48 \mathrm{~h}$, cells were collected and washed with pre-chilled PBS $\left(4^{\circ} \mathrm{C}\right)$. Then, the cells were centrifuged at $1500 \mathrm{rpm}$ for $5 \mathrm{~min}$. After discarding the supernatant, the pellet was resuspended with binding buffer. The cells were then incubated with $5 \mu$ l Annexin V-APC for 15 min in the dark. After fitration of the cell suspension, the analysis of apoptotic cells was performed by FACS can (Becton- Dickinson).

\section{Gene expression profile microarray}

Briefly, A549 cells were seeded in a six-well plate at a density of $4 \times 10^{5}$ cells per well and infected by Lv-shRPS15A and Lv-shCon at MOI of 20, respectively. After 96h, the total RNAs were extracted using TRIzol reagent (Invitrogen, Carlsbad, CA) and were used for cDNA synthesis and labeling, microarray hybridization and then followed by flour-labeled cDNA hybridizing their complements on the chip (Affymetrix, Santa Clara, CA, USA). The resulting localized concentrations of fluorescent molecules were detected and quantified by GeneChip Scanner 3000 (Affymetrix). Finally, the data were analyzed by Expression Console Software (Affymetrix) with default RMA parameters. Data are representative of three separate assays.

\section{Gene ontology annotation and KEGG pathway enrichment analysis}

The gene ontology analysis was performed to functionally annotate the differentially expressed genes according to the Gene Ontology database (http://www.geneontology.org/). The pathway enrichment analysis was performed according to the KEGG (Kyoto Encyclopedia of Genes and 
199 Genomes) database. The Fisher's exact test and $\chi^{2}$ test were applied to classify the significant GO

200 categories and pathways, and the FDR was calculated to correct the $P$-value by multiple 201 comparison tests. A P-value $<0.05$ and an FDG $<0.05$ were set as thresholds to select significant 202 GO categories and pathways.

203

204

\section{Western Blotting assay}

205

206

207

In brief, A549 cells were collected and lysed with precooled lysis buffer after $96 \mathrm{~h}$ of infection.

208

Total protein was extracted from the cells and determined by the BCA method. Protein $(20 \mu \mathrm{g})$

209 was loaded onto a $10 \%$ SDS-PAGE gel. The gel was run at $30 \mathrm{~mA}$ for $2 \mathrm{~h}$ and transferred to poly-vinylidene difluoride membrane (Millipore, Billerica, MA, USA). The resulting membrane

210 was blocked in 5\% non-fat dry milk blocking buffer and then probed with rabbit anti-P21 (1:2,000 dilution; Abcam, Cambridge, MA; Cat. ab7960), mouse anti-TP53I3 (1:500 dilution; Abcam, Cambridge, MA; Cat. ab123917), rabbit anti-SESN2 (1:500 dilution; Abcam, 213 Cambridge, MA; Cat. ab57810) and mouse anti-GAPDH (1:6,000; Santa Cruz Biotechnology, 214 Inc., Sana Cruz, CA) overnight at $4{ }^{\circ} \mathrm{C}$. The protein level of GAPDH was used as a control and 215 detected by an anti-GAPDH antibody. The membrane was washed three times with Tris-buffered 216 saline Tween-20 (TBST), followed by incubation for $2 \mathrm{~h}$ with anti-rabbit and anti-mouse IgG at a

217 1:5,000 dilution (Santa Cruz Biotechnology, Inc.). The membrane was developed using 218 enhanced chemiluminescence (Amersham, UK). Bands on the developed films were quantified 219 with an ImageQuant densitometric scanner (Molecular Dynamics, Sunny-Vale, CA, USA).

\section{Statistic analysis}

222 Data were expressed as mean $\pm \mathrm{SD}$. Comparisons were performed by two-sided independent 223 Student's test, one-way ANOVA analysis and $\chi^{2}$ test using SPSS software for Windows version 22423.0 (SPSS, Chicago, USA). Kaplan-Meier survival curves were plotted and log rank test was 225 done. Statistical significance was accepted when $P<0.05$. All experiments carried out in this 226 study were repeated three independent times. 


\section{Result}

228

229

\section{RPS15A was significantly overexpressed in lung adenocarcinoma tissues}

230

231

We detected the expression of RPS15A protein in a tissue microarray (TMA) of primary lung

232 adenocarcinoma and adjacent normal lung tissue specimens using immunohistochemical staining with an anti-RPS15A antibody. RPS15A immunostaining was primarily detected in cytoplasm. Representative examples of RPS15A protein expression in lung cancer and normal lung samples are shown in Fig. 1a. The immunopositive rates of RPS15A in lung adenocarcinoma and normal lung tissues were $66.7 \%(50 / 75)$ and $42.7 \%$ (32/75), indicating that RPS15A was highly expressed in lung cancer in comparison with adjacent normal tissues, as shown in Table.1 $(P<0.001)$. The correlation between RPS15A expression and different clinical pathological factors in lung adenocarcinoma is shown in Table 2. No significant correlation was found between RPS15A expression and age, grade, TNM stage, tumor size and lymph node metastasis $(P<0.05)$. Kaplan-Meier survival analysis of overall prognosis between patients with higher RPS15A expression and patients with low RPS15A expression was carried out. No significant correlation between RPS15A expression and overall prognosis was found, as shown in Fig $1 \mathrm{~b}$.

\section{Efficacy of lentivirus-mediated RNAi targeting RPS15A}

246

Quantitative real-time PCR was performed to detect the fundamental expression of RPS15 mRNA in several lung cancer cell lines, including lung adenocarcinoma cell lines H1299, A549 and lung squamous cancer cell line SK-MES-1, as well as small cell lung cancer cell line H1688. As shown in Fig. 2a, RPS15A expression levels were quite obvious in the cancer cell lines. Consequently, loss of function assay was applied through RPS15A knockdown. H1299 and A549 cell lines were selected for subsequent studies. To knockdown RPS15A expression, H1299 and A549 cells were infected by the lentiviruses stably expressing RPS15A-specific shRNA (LvshRPS15A). Lentivirus expressing negative shRNA (Lv-shCon) was used as negative control. More than $80 \%$ of GFP-expressing cells were observed under fluorescence microscope after $72 \mathrm{~h}$ (Fig. 2b). The silencing effect of lentivirus mediated RPS15A RNAi on RPS15A expression in H1299 and A549 cells was examined through real-time PCR and Western Blotting assay. The expression level of RPS15A in the LV-shRPS15A infected cells was significantly lower than that in the Lv-shCon infected cells (Fig. 2c, 2d). Therefore, these data indicated the high efficacy of lentivirus mediated RPS15A silence in lung cancer cells.

261 
264 To investigate the role of RPS15A in the proliferation of lung cancer cells, the proliferative 265 abilities of Lv-shCon and Lv-shRPS15A infected H1299 and A549 cells was determined by 266 MTT assay. During the 120 hour incubation period, the growth of Lv-shRPS15A infected cells 267 was significantly slower than that of Lv-shCon infected cells at the time points of $48 \mathrm{~h}, 72 \mathrm{~h}, 96$ $268 \mathrm{~h}$ and $120 \mathrm{~h}$ (Fig. 3a and 3b). To investigate the effect of RPS15A downregulation on tumor 269 formation, colony formation assay was performed. Quantitative analysis of colonies showed that 270 after incubation for 10 days, the number of colonies in Lv-shRPS15A infected cells was 271 significantly lower than that in the Lv-shCon infected cells (Fig. 3c, 3d, 3e and 3f). Therefore, 272 the low colony-forming efficiency of Lv-shRPS15A infected H1299 and A549 cells 273 demonstrated that RPS15A silencing inhibited the colony forming ability of lung cancer cells in 274 vitro.

275

\section{RPS15A silence triggered cell apoptosis in vitro}

277

278

To determine whether RPS15A knockdown induces apoptosis in H1299 and A549 cells, 279 Annexin V-APC staining was performed and the percentage of apoptotic cells was detected by 280

281

282

283 284

285

286

287

288

289

290

291

292

293

294

295

296

297

298

299

\section{RPS15Aknockdown activated P53 signaling pathway in vitro}

To explore the potential downstream mechanisms underlying the actions of RPS15A silencing, a cDNA microarray assay was performed to compare the differential gene expression profiles between Lv-shRPS15A infected and Lv-shCON infected A549 cancer cells. Microarray analysis demonstrated that 885 genes were upregulated and 566 genes were downregulated significantly in Lv-shRPS15A infected cancer cells when compared to Lv-shCON infected cells. To further classify the function of these genes, GO analysis was carried out. The most significant GO categories of biological processes included protein, biopolymer and cellular macromolecule metabolic processes. Of these genes, the most enriched GO in terms of molecular function involved DNA binding, cation and ion binding and enzyme regulator activity. KEGG pathway enrichment analysis revealed that certain signaling pathways participated in the tumor inhibition induced by RPS15A knockdown, of which P53 signaling pathway members were most evidently annotated. The top 20 significantly perturbed pathways are listed in Table 3 . To confirm the P53 pathway activation, the expressions of key factors of P53 signaling pathway were determined 
300 through western blotting. Consistent with microarray results, P21 and TP53I3 expressions were 301 upregulated by RPS15A knockdown, while SESN2 expression was downregulated, as shown in 302 Fig.4e.

303 
305

306

307

308

309

310

311

312

313

314

315

316

317

318

319

320

321

322

323

324

325

326

327

328

329

330

331

332

333

334

335

336

337

338

339

340

341

\section{Discussion}

Therapeutic drugs targeting cancer-related molecules can specifically inhibit malignant cells, causing minimal adverse off target reactions because of the well-defined mechanisms (Chen et al. 2013). Therefore, identification of molecular factors responsible for pulmonary carcinogenesis and elucidation of their underlying mechanisms of proliferation are urgently needed for novel therapeutic targets.

Earlier genetic studies in zebrafish suggested that many ribosomal protein genes are haploidsufficient suppressor gene that are highly related to tumorigenesis (Amsterdam et al. 2004). Ribosomal proteins are abundant in most cells and well known for their functional role in the assembly of ribosomal subunits at the early stages of translation. In addition, ribosomal proteins have been found to regulate cell proliferation, apoptosis, DNA repair and gene transcription. Currently, ribosomal proteins are emerging as novel regulators of cancer cell growth, whose mutations and changes of expression level are highly relevant to human malignancies. RPS15A, as a component of the $40 \mathrm{~S}$ ribosomal subunit, has been found to facilitate the binding of capped mRNA to the ribosomal subunit $40 \mathrm{~S}$ in translation initiation. Furthermore, it has been reported that downregulation of its mRNA expression in hepatocellular carcinoma cells significantly inhibits tumor growth, suggesting that RPS15A may play a role in human carcinogenesis. Yet, up to now, little is known about its function in NSCLC cells.

To determine the expression levels of RPS15A in lung adenocarcinoma tissues, TMAs was performed and revealed that RPS15A was highly expressed in lung cancer tissue. Therefore, we hypothesized that RPS15A may play an important role in the proliferation of lung cancer. In this regard, a lentivirus-mediated RNAi system was applied to inhibit RPS15A mRNA expression in human lung adenocarcinoma H1299 and A549 cells in vitro. Lentivirus expressing RPS15Aspecific shRNA was constructed and used to infect H1299 and A549 cells. The efficiency of lentivirus-induced silencing of endogenous RPS15A was confirmed by qPCR and western blotting assay. To determine the impact of RPS15A knockdown on the lung cancer growth in vitro, an MTT assay and colony formation assay were carried out. As a result, downregulation of RPS15A expression greatly impaired the proliferation and colony-forming ability of H1299 and A549 cells. Furthermore, flow cytometry analysis data showed that RPS15A silencing induced apoptosis as characterized by the prominent presence of apoptotic cancer cells.

To elucidate the downstream mechanisms underlying RPS15A silence in lung cancer, we carried out a human whole genome oligo microarray and KEGG pathway enrichment analysis. The data revealed that the P53 signaling pathway was activated significantly in Lv-shRPS15A infected 
342 A549 cells. The P53 pathway has been well known for its anticancer function through initiating 343 apoptosis, cell cycle arrest, maintaining genomic stability, angiogenesis inhibition etc (Levine \& 344 Oren 2009). To confirm the P53 signaling activation, we determined the expression of P21, 345 TP53I3 and SESN2 by using western blotting. P21(P21), which is tightly controlled by the tumor 346 suppressor protein p53, is a potent cyclin-dependent kinase inhibitor that binds to cyclin-CDK2 347 or -CDK4 complexes, thus functioning as a regulator of cell cycle progression at G1 (el-Deiry et 348 al. 1993).TP53I3 is induced by the tumor suppressor p53 and is thought to be involved in p53mediated cell death (Polyak et al. 1997). SESN2 is also known for its function in the regulation of cell growth and survival in cellular response to different stress conditions (Hay 2008).

353

354

355

356

357

358

359

360

361

362

363

364

365

366

367

368

369

370

371

372

373

374

375

376

377

378

379

380

381
Ribosomal proteins are often classified as cell growth associated molecules due to their key role in protein synthesis (Eid et al. 2014; Lian et al. 2004; Wang et al. 2014). Our finding that RPS15A downregulation inhibits NSCLC cell growth is also supported by previous studies that RPS15A knockdown also inhibits hepatic cancer cell growth (Xu et al. 2014). Like other ribosomal proteins, RPS15A has been found to involve extra-ribosomal functions in cell growth, apoptosis and cell cycle transition. In this study, our findings that RPS15A induced apoptosis and cell cycle phase arrest in lung cancer A549 cells further supported that P53 signaling is critical for the regulation of apoptosis and cell cycle transition. However, to date, the issue of whether and how RPS15A interacts with other regulators remains poorly studied, and further investigation is warranted to elucidate the detailed mechanisms underlying the action of RPS15A. Taken together, our study demonstrated that RPS15A might serve as an upstream modulator of P53 signaling pathway.

In conclusion, RPS15A expression was increased in tumor tissues. Furthermore, the knockdown of RSP15A inhibited cancer cell growth and induced apoptosis in the cancer cells. Gene expression profile microarray also revealed that the P53 signaling pathway was activated in LvshRPS15A-infected cancer cells. Therefore, our findings demonstrate that RPS15A is a novel oncogene in non-small cell lung cancer and may be a potential therapeutic target in lung cancer.

\section{References}

Amsterdam A, Sadler KC, Lai K, Farrington S, Bronson RT, Lees JA, and Hopkins N. 2004. Many ribosomal protein genes are cancer genes in zebrafish. PLoS Biol 2:E139.

Butnor KJ, and Beasley MB. 2007. Resolving dilemmas in lung cancer staging and histologic typing. Arch Pathol Lab Med 131:1014-1015.

Chan YL, Olvera J, Paz V, and Wool IG. 1994. The primary structure of rat ribosomal protein S15a. Biochem Biophys Res Commun 200:1498-1504.

Chen X, Liu Y, Roe OD, Qian Y, Guo R, Zhu L, Yin Y, and Shu Y. 2013. Gefitinib or erlotinib as maintenance therapy in patients with advanced stage non-small cell lung cancer: a systematic review. PLoS One 8:e59314.

Eid R, Sheibani S, Gharib N, Lapointe JF, Horowitz A, Vali H, Mandato CA, and Greenwood MT. 2014. Human 
ribosomal protein $L 9$ is a Bax suppressor that promotes cell survival in yeast. FEMS Yeast Res 14:495-507. el-Deiry WS, Tokino T, Velculescu VE, Levy DB, Parsons R, Trent JM, Lin D, Mercer WE, Kinzler KW, and Vogelstein B. 1993. WAF1, a potential mediator of $\mathrm{p} 53$ tumor suppression. Cell 75:817-825.

Hanahan D, and Weinberg RA. 2011. Hallmarks of cancer: the next generation. Cell 144:646-674.

Hay N. 2008. p53 strikes mTORC1 by employing sestrins. Cell Metab 8:184-185.

Hensing T, Chawla A, Batra R, and Salgia R. 2014. A personalized treatment for lung cancer: molecular pathways, targeted therapies, and genomic characterization. Adv Exp Med Biol 799:85-117.

Jemal A, Bray F, Center MM, Ferlay J, Ward E, and Forman D. 2011. Global cancer statistics. CA Cancer J Clin 61:6990.

Lavoie C, Tam R, Clark M, Lee H, Sonenberg N, and Lasko P. 1994. Suppression of a temperature-sensitive cdc33 mutation of yeast by a multicopy plasmid expressing a Drosophila ribosomal protein. I Biol Chem 269:14625-14630.

Lee SB, Kwon IS, Park J, Lee KH, Ahn Y, Lee C, Kim J, Choi SY, Cho SW, and Ahn JY. 2010. Ribosomal protein S3, a new substrate of Akt, serves as a signal mediator between neuronal apoptosis and DNA repair. J Biol Chem 285:29457-29468.

Levine AJ, and Oren M. 2009. The first 30 years of p53: growing ever more complex. Nat Rev Cancer 9:749-758.

Li C, Ge M, Yin Y, Luo M, and Chen D. 2012. Silencing expression of ribosomal protein L26 and L29 by RNA interfering inhibits proliferation of human pancreatic cancer PANC-1 cells. Mol Cell Biochem 370:127-139.

Lian Z, Liu J, Li L, Li X, Tufan NL, Wu MC, Wang HY, Arbuthnot P, Kew M, and Feitelson MA. 2004. Human S15a expression is upregulated by hepatitis B virus $X$ protein. Mol Carcinog 40:34-46.

Linder P, and Prat A. 1990. Baker's yeast, the new work horse in protein synthesis studies: analyzing eukaryotic translation initiation. Bioessays 12:519-526.

Miller YE. 2005. Pathogenesis of lung cancer: 100 year report. Am J Respir Cell Mol Biol 33:216-223.

Nishiura H, Zhao R, Chen J, Taniguchi K, and Yamamoto T. 2013. Involvement of regional neutrophil apoptosis promotion by ribosomal protein S19 oligomers in resolution of experimental acute inflammation. Pathol Int 63:581-590.

Nosrati N, Kapoor NR, and Kumar V. 2014. Combinatorial action of transcription factors orchestrates cell cycledependent expression of the ribosomal protein genes and ribosome biogenesis. FEBS J.

Paleari L, Russo P, Cesario A, and Granone P. 2008. Factors predicting poor survival after resection of stage IA nonsmall cell lung cancer. J Thorac Cardiovasc Surg 136:241-242.

Polyak K, Xia Y, Zweier JL, Kinzler KW, and Vogelstein B. 1997. A model for p53-induced apoptosis. Nature 389:300305.

Schaap PJ, de Groot PW, Muller Y, van Griensven L, and Visser J. 1995. Molecular cloning and sequence of the cytoplasmic ribosomal protein S15a gene from Agaricus bisporus. Exp Mycol 19:160-162.

Wang L, Luo J, Nian Q, Xiao Q, Yang Z, and Liu L. 2014. Ribosomal protein S14 silencing inhibits growth of acute myeloid leukemia transformed from myelodysplastic syndromes via activating p53. Hematology 19:225231.

Xu M, Wang Y, Chen L, Pan B, Chen F, Fang Y, Yu Z, and Chen G. 2014. Down-regulation of ribosomal protein S15A mRNA with a short hairpin RNA inhibits human hepatic cancer cell growth in vitro. Gene 536:84-89.

Yang M, Sun H, Wang H, Zhang S, Yu X, and Zhang L. 2013. Down-regulation of ribosomal protein L22 in non-small cell lung cancer. Med Oncol 30:646. 
425 Figure Legend

426

427 Figure 1. Immunostaining of RPS15A in lung adenocarcinoma and adjacent normal tissues with 428 tissue microarray. (a) Three representative cases with different expression status of RPS15A, 429 ranging from negative, mild and strong expression were taken at $100 \times$ and $400 \times$ magnification 430 in lung cancer and normal tissues. (b) Kaplan-Meier survival analysis of overall prognosis 431 between patients with higher RPS15A expression and patients with low RPS15A expression. 432 Log-rank test was used to statistically calculate the difference.

433

434 435

436

437

438

439

440

441

442

443

444

445

446

447

448

449

450

451

452

453

454

455

456

457

458

Figure 2. Lentiviral mediated RPS15A downregulation. (a) Relative RPS15A mRNA level in lung cancer cell lines (H1299, A549, SK-MES-1, H1688 and H1975). (b) Fluorescence photomicrographs of H1299 and A549 cells 72h after lentivirus infection. (c, d) RPS15A mRNA and protein expressions were dramatically downregulated in LV-shRPS15A infected cells evidenced by real-time PCR and Western blotting assay. $* * \mathrm{P}<0.01$ versus Lv-shCon.

Figure 3. The proliferation of H1299 and A549 cells was inhibited after LV-shRPS15A infection determined by MTT assay. (a, b). The colony formation abilities of H1299 and A549 cells were determined by colony formation assay after Lv-shRPS15A infection. (c, d) Images of colonies and statistical analysis of the number of colonies. (e, f) Images of colonies recorded under microscope. $* \mathrm{P}<0.05, * * \mathrm{P}<0.01$ versus $\mathrm{Lv}$-shCon.

Figure 4. RPS15A knockdown induced apoptotic cells were determined by flow cytometry analysis after Annexin V-APC staining. (a, b) Histograms of FACS analysis. (c, d) Percentage of apoptotic cells. $* * \mathrm{P}<0.01$ versus Lv-shCon. (e) Key factors of P53 signaling pathway, such as P21, TP53I3 and SESN2, were examined in LV-shRPS15A infected A549 cells by using western blotting method. The protein level of GAPDH was employed as a control.

Table 1. RPS15A expression in 75 lung adenocarcinoma and adjacent normal tissue specimens

Table 2. Correlation between RPS15A expression and clinicopathological factors in 75 lung adenocarcinoma patients specimens.

Table 3. KEGG pathway enrichment analysis revealed that P53 signaling pathway members were most evidently annotated. Top 20 significantly perturbed pathways are listed. 


\section{1}

Immunostaining of RPS15A with tissue microarray

Immunostaining of RPS15A in lung adenocarcinoma and adjacent normal tissues with tissue microarray. (a) Three representative cases with different expression status of RPS15A, ranging from negative, mild and strong expression were taken at $100 \times$ and $400 \times$ magnification in lung cancer and normal tissues. (b)Kaplan-Meier survival analysis of overall prognosis between patients with higher RPS15A expression and patients with low RPS15A expression. Log-rank test was used to statistically calculate the difference. 
A

Normal tissue

Lung cancer

Lung cancer no RPS15A low RPS15A strong RPS15A

$100 x$
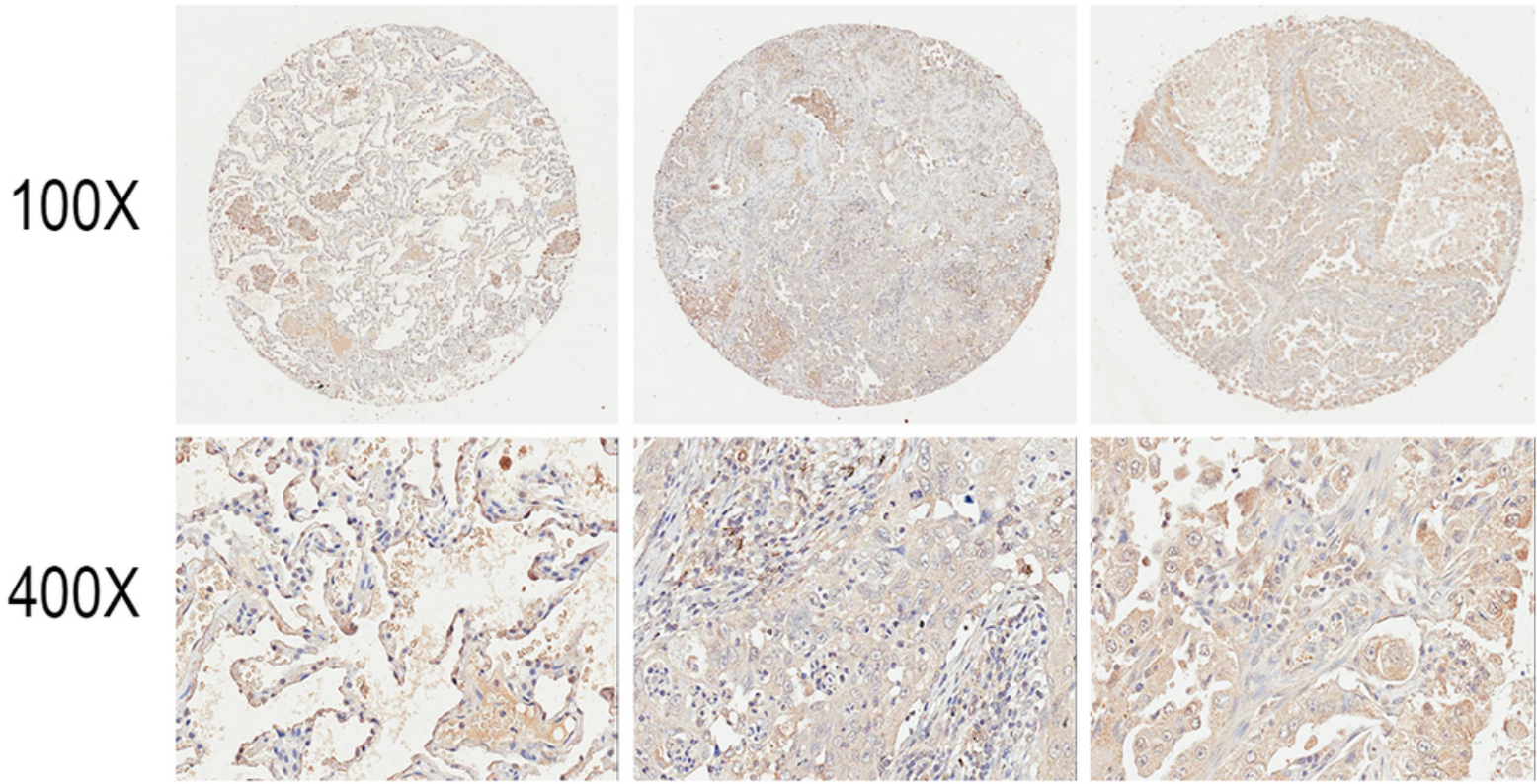

B

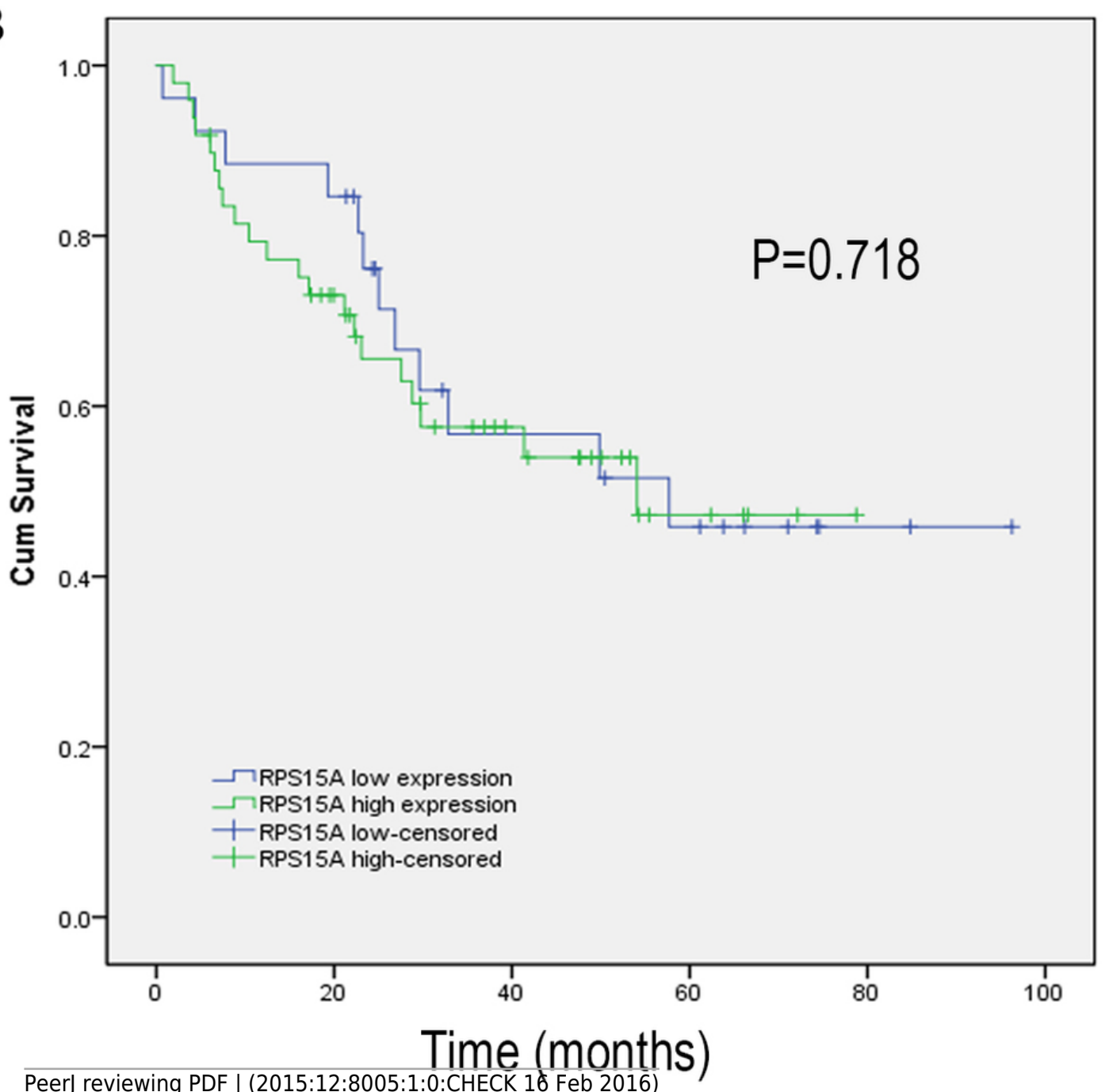

Peer] reviewing PDF | (2015:12:8005:1:0:CHECK 16 Feb 2016) 
2

Lentiviral mediated RPS15A downregulation.

Lentiviral mediated RPS15A downregulation. (a) Relative RPS15A mRNA level in lung cancer cell lines (H1299, A549, SK-MES-1, H1688 and H1975). (b) Fluorescence photomicrographs of H1299 and A549 cells 72h after lentivirus infection. (c, d) RPS15A mRNA and protein expressions were dramatically downregulated in Lv-shRPS15A infected cells evidenced by real-time PCR and Western blotting assay. ${ }^{*} \mathrm{P}<0.01$ versus LV-shCon. 


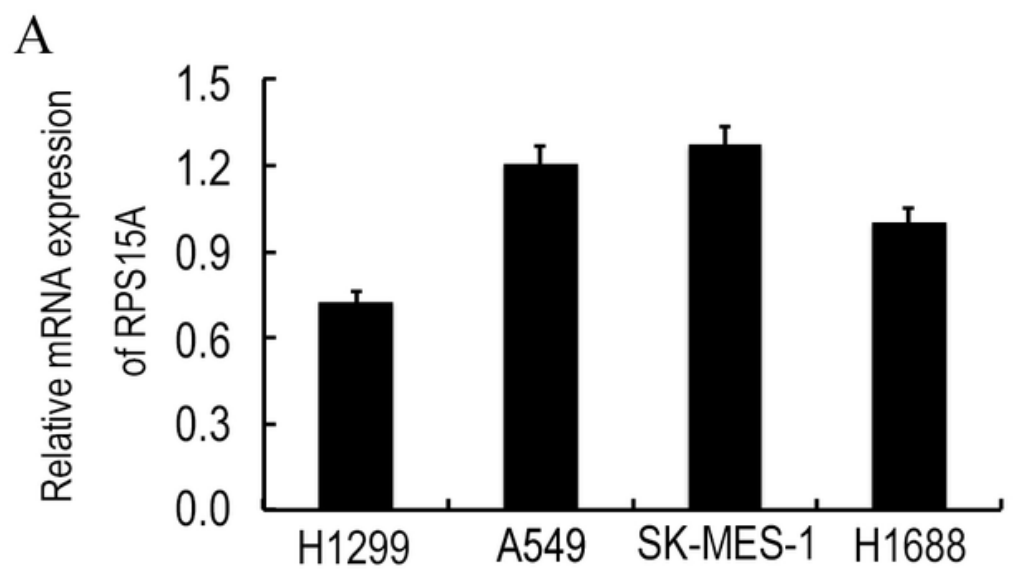

B

Lv-shCon

Lv-shRPS15A

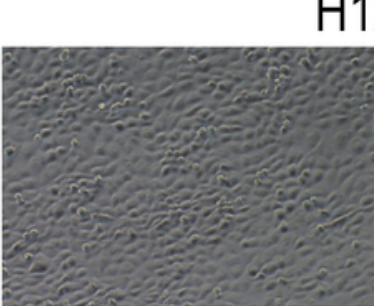

H1299
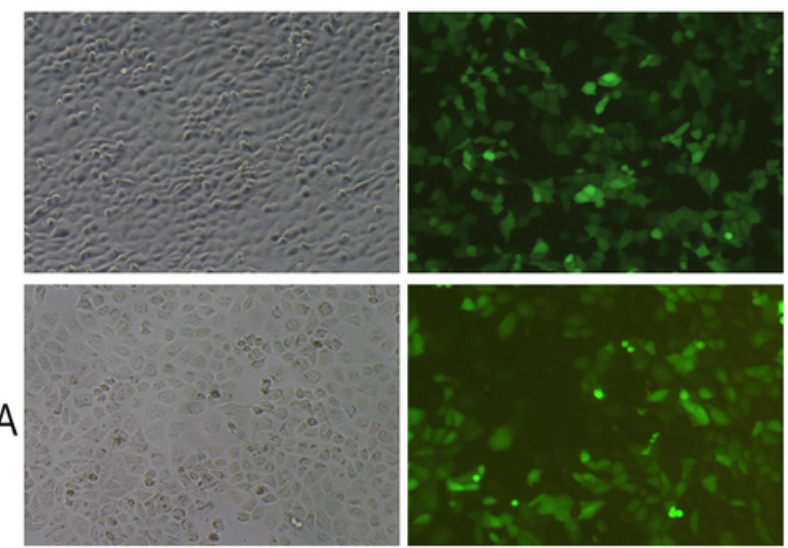

C

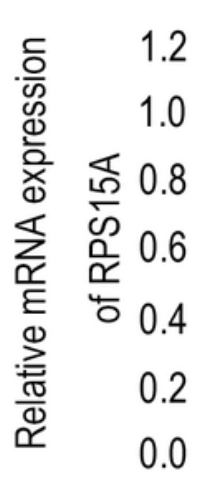

D

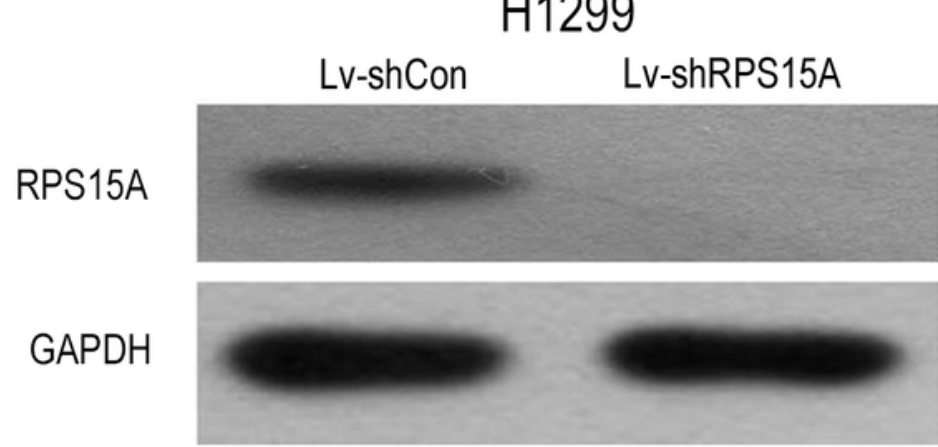

A549

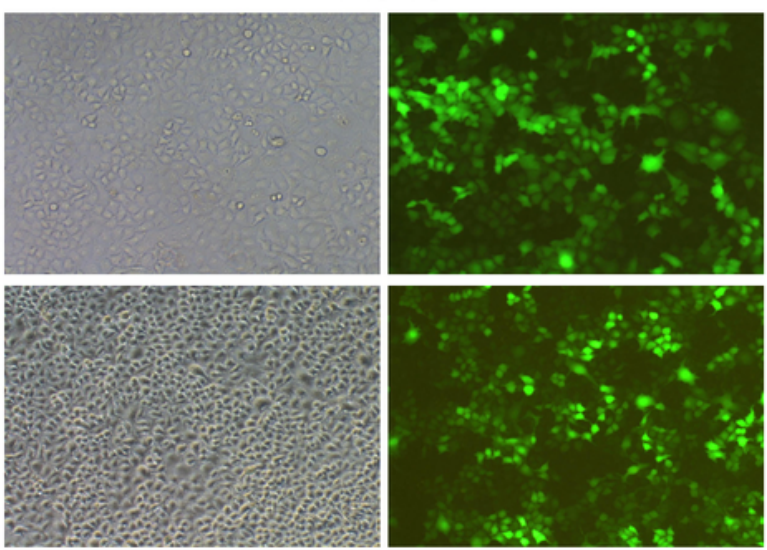

A549

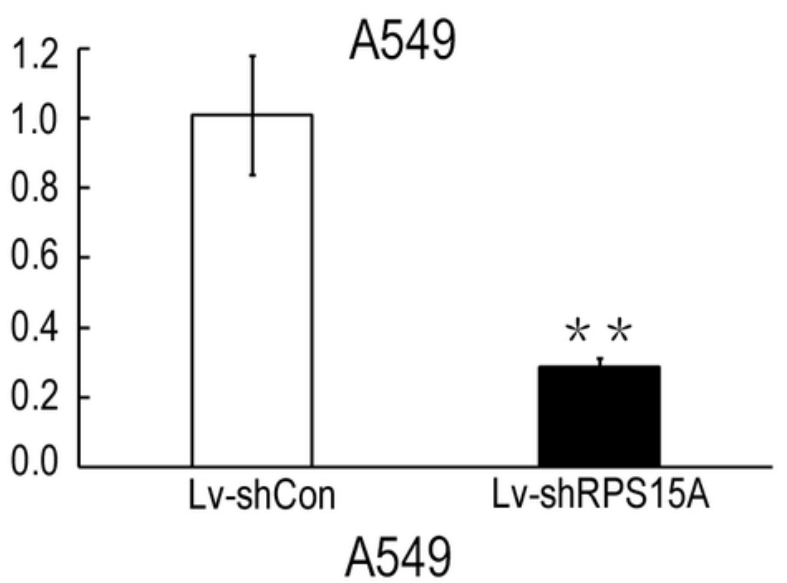

Lv-shCon

Lv-shRPS15A

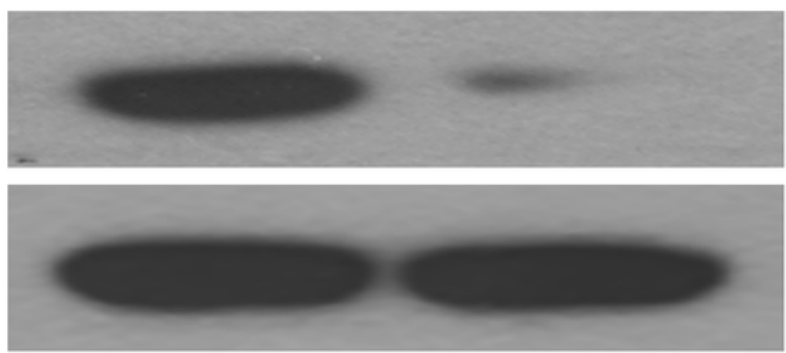


3

The proliferation of $\mathrm{H} 1299$ and $\mathrm{A} 549$ cells

The proliferation of H1299 and A549 cells was inhibited after Lv-shRPS15A infection determined by MTT assay. $(a, b)$. The colony formation abilities of H1299 and A549 cells were determined by colony formation assay after Lv-shRPS15A infection. (c, d) Images of colonies and statistical analysis of the number of colonies. (e, f) Images of colonies recorded under microscope. $* \mathrm{P}<0.05, * * \mathrm{P}<0.01$ versus LV-shCon. 

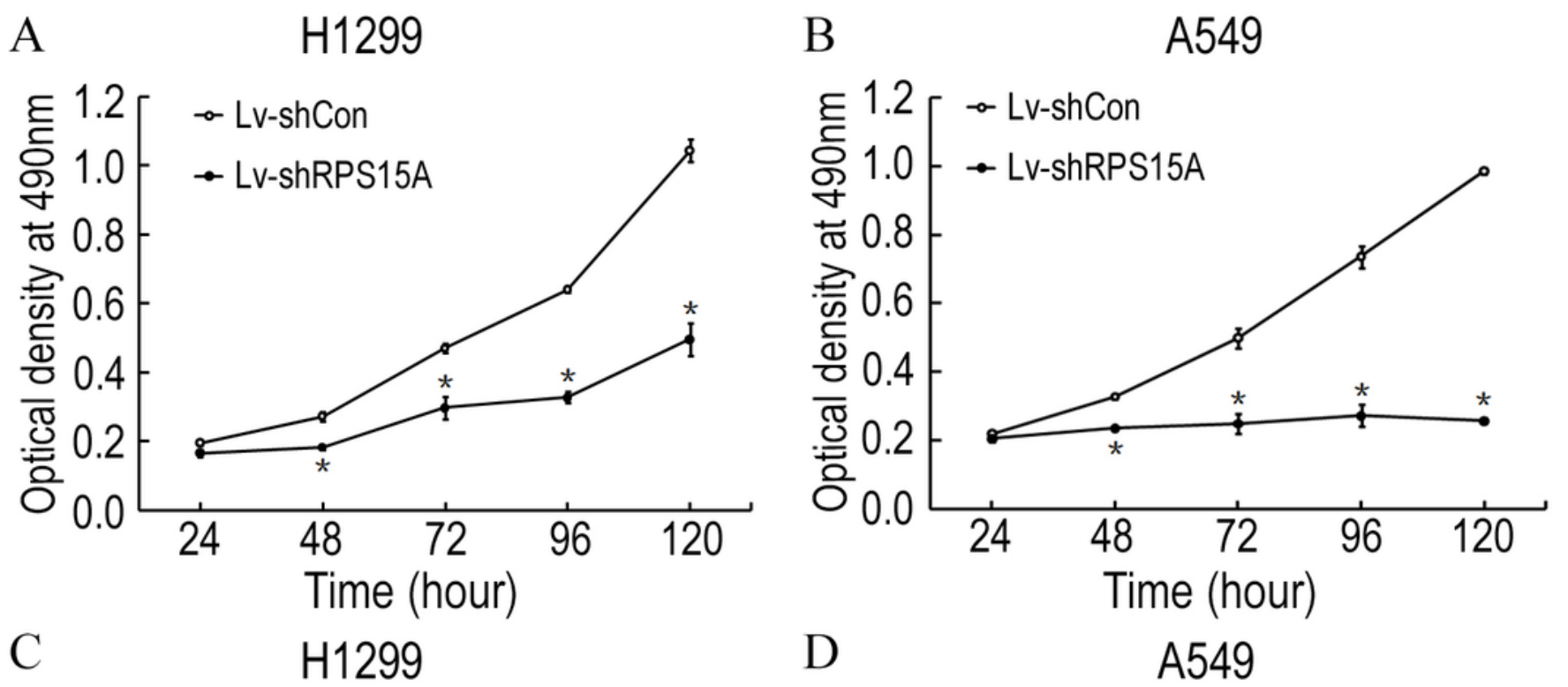

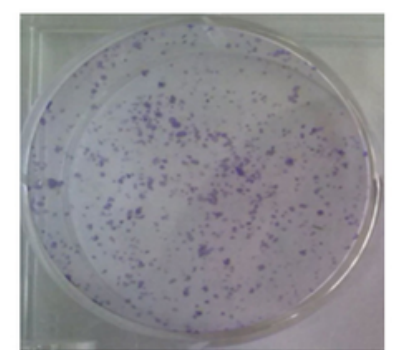

LV-shCon

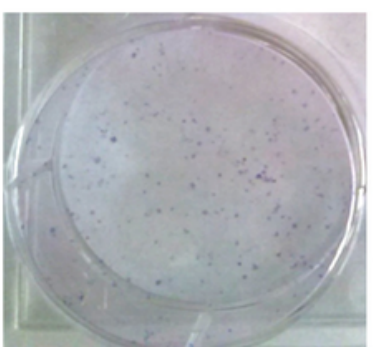

Lv-shRPS15A

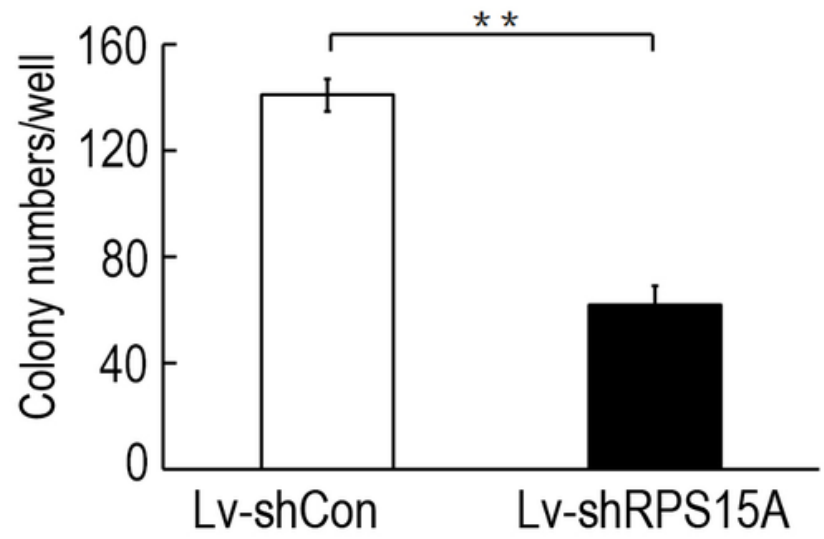

E

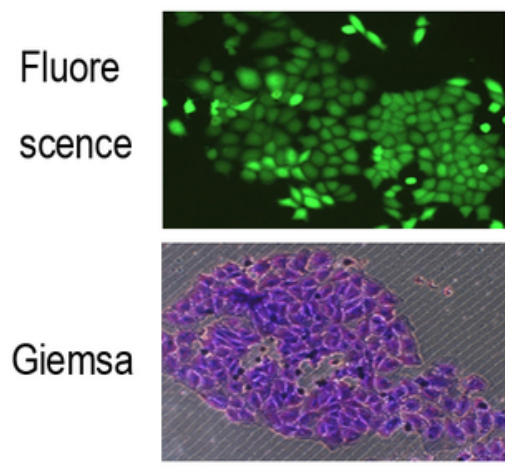

LV-shCon
H1299
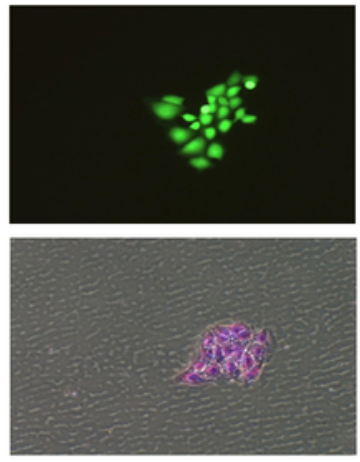

Lv-shRPS15A

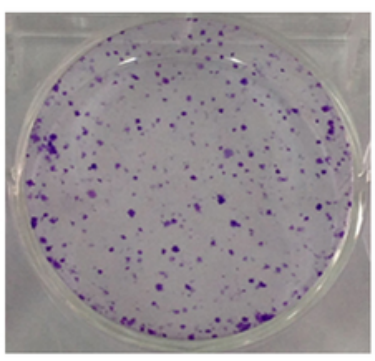

LV-shCon

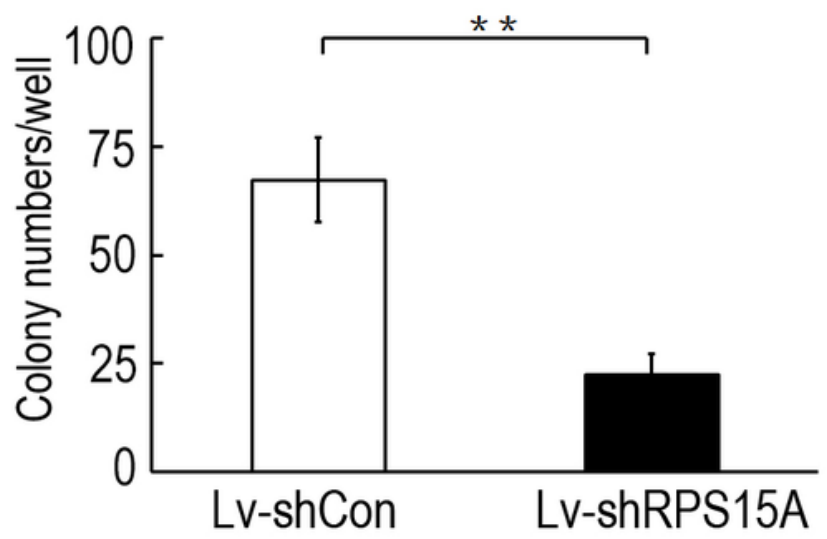

F

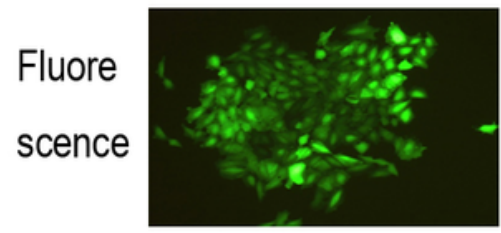

Giemsa

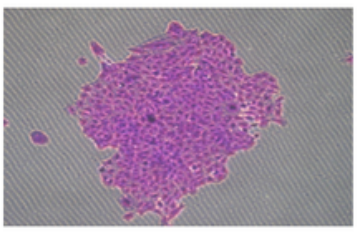

Lv-shCon

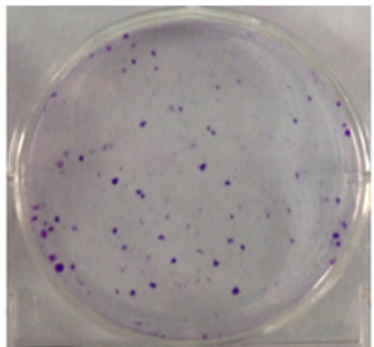

Lv-shRPS15A

A549
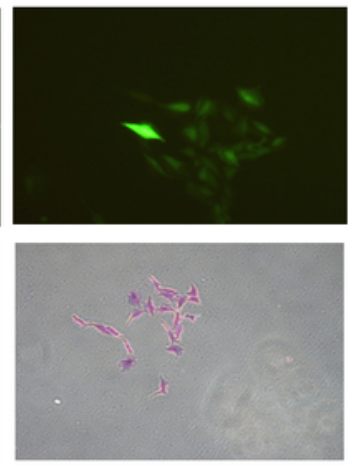

Lv-shRPS15A 


\section{4}

RPS15A knockdown induced apoptotic cells

RPS15A knockdown induced apoptotic cells were determined by flow cytometry analysis after Annexin V-APC staining. (a, b) Histograms of FACS analysis. (c, d) Percentage of apoptotic cells. ${ }^{* * P}<0.01$ versus Lv-shCon. (e) Key factors of P53 signaling pathway, such as P21,TP5313and SESN2, were examined in Lv-shRPS15A infected A549 cells by using western blotting method. The protein level of GAPDH was employed as a control. 

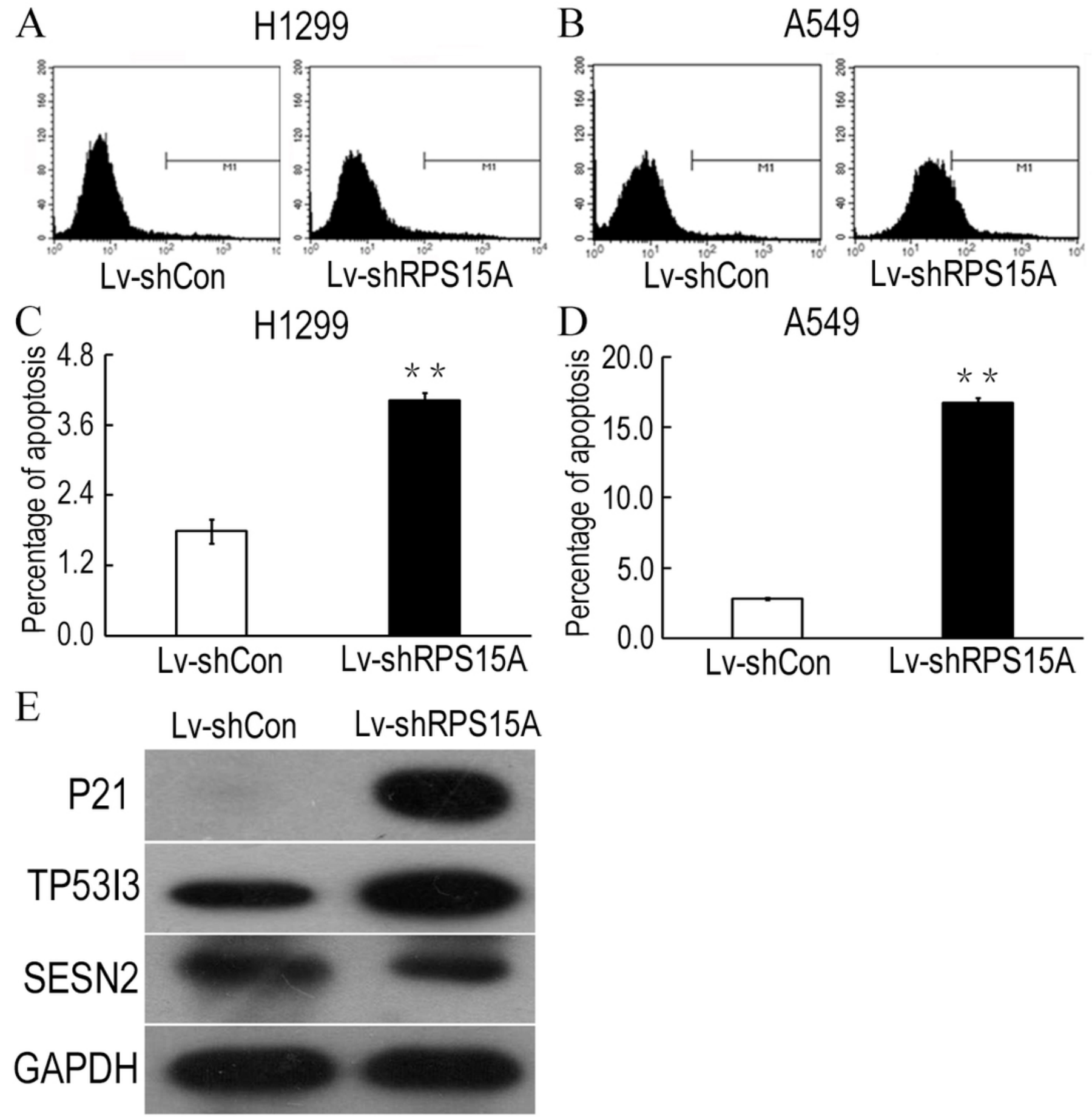

D

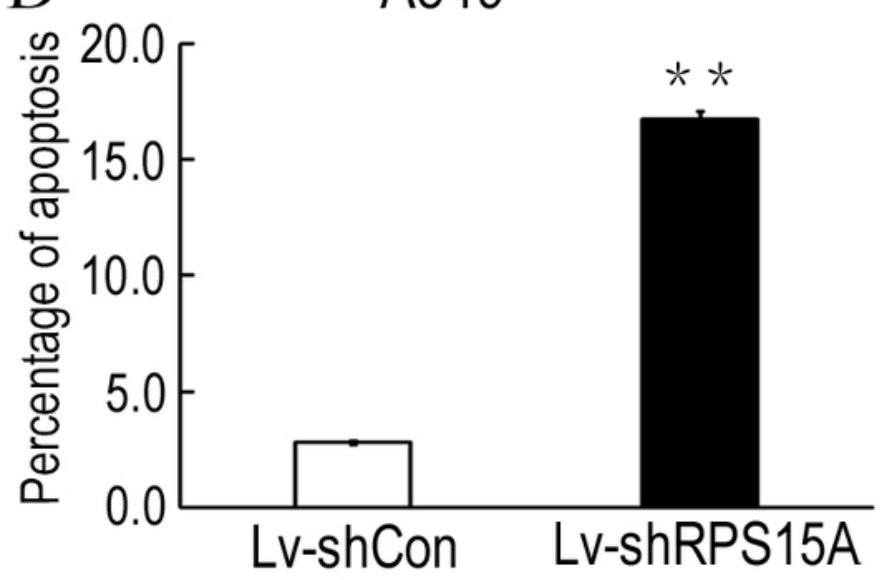




\section{Table 1 (on next page)}

RPS15A expression in 75 lung adenocarcinoma and adjacent normal tissue specimens . 
1 Table 1 RPS15A expression in 75 lung adenocarcinoma and adjacent normal tissue specimens $2 * \mathrm{P}$ values were obtained with the $\chi^{2}$ test, $\mathrm{P}<0.001$.

\begin{tabular}{|c|c|c|c|c|c|c|}
\hline \multirow[t]{2}{*}{ Histological types } & \multirow[t]{2}{*}{ Number } & \multicolumn{4}{|c|}{ RPS15A expression } & \multirow[t]{2}{*}{$\mathbf{p}^{*}$} \\
\hline & & - & + & ++ & +++ & \\
\hline Cancer & 75 & 11 & 19 & 15 & 35 & 0.000 \\
\hline Normal tissue & 75 & 5 & 38 & 26 & 6 & \\
\hline
\end{tabular}




\section{Table 2 (on next page)}

Correlation between RPS15A expression and clinicopathological factors in 75 lung adenocarcinoma patients specimens. 
1 Table 2 Correlation between RPS15A expression and clinicopathological factors in 75 lung

2 adenocarcinoma tissue specimens

\begin{tabular}{|c|c|c|c|c|}
\hline \multirow[t]{2}{*}{ Variables } & \multirow[t]{2}{*}{ All patients } & \multicolumn{2}{|c|}{ RPS15A expression } & \multirow[t]{2}{*}{$\mathbf{p}^{*}$} \\
\hline & & Negative & Positive & \\
\hline Total & 75 & 26 & 49 & \\
\hline \multicolumn{5}{|l|}{$\operatorname{Age}(y)$} \\
\hline$\leq \mathbf{6 0}$ & 48 & 14 & 25 & 1.000 \\
\hline$>60$ & 27 & 12 & 24 & \\
\hline \multicolumn{5}{|l|}{ Gender } \\
\hline Male & 43 & 19 & 24 & 0.053 \\
\hline Female & 32 & 7 & 25 & \\
\hline \multicolumn{5}{|c|}{ TNM stage } \\
\hline I II & 47 & 20 & 36 & 0.788 \\
\hline III IV & 28 & 6 & 13 & \\
\hline \multicolumn{5}{|c|}{ Tumor size } \\
\hline$\leq 3 \mathrm{~cm}$ & 8 & 1 & 7 & 0.249 \\
\hline$>3 \mathrm{~cm}$ & 67 & 25 & 42 & \\
\hline \multicolumn{5}{|c|}{$\begin{array}{l}\text { Lymph node } \\
\text { metastasis }\end{array}$} \\
\hline Yes & 28 & 8 & 20 & 0.458 \\
\hline No & 47 & 18 & 29 & \\
\hline
\end{tabular}

$3 * \mathrm{P}$ values were obtained with the $\chi^{2}$ test 


\section{Table 3(on next page)}

KEGG pathway enrichment analysis revealed that P53 signaling pathway members were most evidently annotated. Top 20 significantly perturbed pathways are listed. 
Table 3 Top 20 highly expressed signaling pathways induced by RPS15A silencing

$-\lg P$

p53 signaling pathway NOD-like receptor signaling pathway Complement and coagulation cascades Steroid biosynthesis Pathways in cancer ErbB signaling pathway Biosynthesis of unsaturated fatty acids Ribosome

Neurotrophin signaling pathway MAPK signaling pathway Apoptosis

Adipocytokine signaling pathway Hypoxia and p53 in the Cardiovascular system Aminoacyl-tRNA biosynthesis Cell cycle Purine metabolism Focal adhesion HIV-I Nef: negative effector of Fas and TNF Small cell lung cancer Chemokine signaling pathway
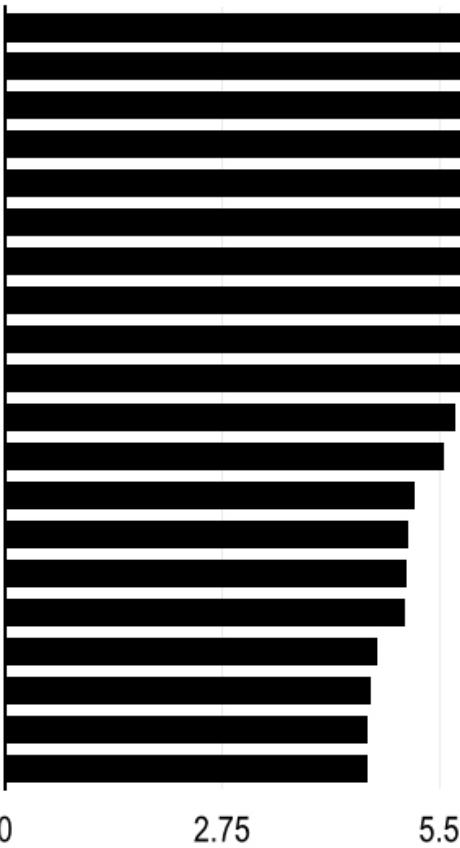

\begin{tabular}{l}
5.5 \\
\hline
\end{tabular} 